\title{
Modeling Macrosegregation during Direct-Chill Casting of Multicomponent Aluminum Alloys
}

\author{
Q. DU, D.G. ESKIN, and L. KATGERMAN
}

\begin{abstract}
A macrosegregation model for direct chill casting of multicomponent aluminum alloys is implemented using the macroscopic transfer model, microsegregation model, and phase diagram calculation module, and applied to an $\mathrm{Al}-\mathrm{Cu}-\mathrm{Mg}$ alloy. The phase diagram calculation module is based on the TQ-Interface of CALPHAD software THERMO-CALC and the mapping technique initially proposed by Dore et al. This mapping technique is modified in arranging the mapping axes where the tabulation is performed to increase the access efficiency. This strategy provides a practical solution for quick access to phase diagram data in modeling macrosegregation of multicomponent alloys. It is found from our simulation that the contribution of each of the solute elements to the solutal buoyancy affects the final segregation pattern. The appropriate choice of the solidification path is important for the shrinkage-induced macrosegregation. The model is applied to a real direct-chill (DC) casting experiment and a reasonable semiquantitative agreement with experimental data has been obtained, though the model does not take into account the possible contribution of floating grains and exudation.
\end{abstract}

DOI: $10.1007 / \mathrm{s} 11661-006-9042-0$

(C) The Minerals, Metals \& Materials Society and ASM International 2007

\section{INTRODUCTION}

MACROSEGREGATION modeling became a working instrument thanks to the pioneering work of Flemings et al. in the 1960s. ${ }^{[1]}$ The main mechanism behind macrosegregation is now well understood, i.e., the transport of segregated alloying elements at the scale of a casting by the relative movement of liquid and solid phases. The most widely used macrosegregation models are single domain methods, based on either volume averaging technique ${ }^{[2,3]}$ or mixing theory, ${ }^{[4]}$ in which the rigid solid phase usually is treated as highly viscous fluid (pseudo-fluid concept). Recently, Beckermann ${ }^{[5]}$ gave a good review of macrosegregation modeling, emphasizing the applications of macrosegregation models in various industrial casting processes.

Our research work is intended to enable the application of macrosegregation modeling in the industrial direct-chill (DC) casting process by implementing an efficient method to access the phase diagram data and by taking full advantage of the features provided by the commercial CFD software, CFX-5,* such as unstruc-

* CFX is a trademark of Ansys, Inc., Canonsburg, PA.

tured meshing and parallel computing.

Over the last decade, several articles have been published with an attempt to extend the application of macrosegregation models to ternary alloys, ${ }^{[6,7,8]}$ because

Q. DU, Postdoctor and D.G. ESKIN, Senior Scientist are with the Netherlands Institute for Metals Research, 2628CD Delft, the Netherlands. Contact e-mail: q.du@nimr.nl L. KATGERMAN, Professor, is with the Delft University of Technology, 2628CD Delft, the Netherlands.

Manuscript submitted April 20, 2006. the local solidification path can be changed dramatically by the addition of alloying elements. Krane and Incropera concluded that the continuum mixture equations of ternary alloys were essentially the same as those for a binary alloy, differing only in the addition of a second species Eq. ${ }^{[6]}$ They also found in their simulation results on the lever-rule type solidification of a ternary $\mathrm{Pb}-\mathrm{Sb}-\mathrm{Sn}$ alloy that macrosegregation of the solutes was altered significantly from the familiar patterns of the binary systems when the alloy composition was close to the monovariant lines of the ternary phase diagram. If the secondary solidification occurred deep in the mushy zone, the resulting microsegregation had only small effects on the final redistribution of solute. ${ }^{[7]}$ The addition of a second alloying element may also change the flow velocity through the solutal buoyancy term, and therefore may alter the final macrosegregation pattern. It remains, however, unclear if this effect is important in DC casting of aluminum alloys.

The extension to multicomponent alloys with a "real" phase diagram is of great industrial interest but requires a special strategy. The difficulty in solving the set of equations for multicomponent alloys originates from the demand to have an easy and quick access to the phase diagram information. To solve this problem, indirect coupling with a CALPHAD type of software, namely, the mapping technique, has been implemented and successfully applied to describe Al-Mg-Si solidification paths. ${ }^{[9]}$ In this approach, the information related to each forming solid phase, including its liquidus and partition coefficients, is tabulated into a file, and a specific phase diagram module is provided to deduce equilibrium phase fractions and concentrations under a given temperature/composition condition. This strategy can be improved in macrosegregation simulation of DC casting of aluminum alloys based on the following considerations. 
(1) Although solidification proceeds in a nonequilibrium way, local equilibrium is often assumed at the solid-liquid interface under the solidification conditions experienced in DC casting. Microscopic models can be built on the data about the equilibrium state at a given temperature and the average concentration.

(2) The types and fractions of the secondary phases are not important except for their influence on the liquid concentration and fraction. All of the solid phases can be treated as a single phase, which is a reasonable approximation for the mixture momentum and enthalpy according to Reference 6, if the solid phase densities and specific heats are close to one another.

In Reference 9, a mapping file is created in terms of the liquidus compositions and partition coefficients, and then a quite CPU-time-consuming procedure for complex multicomponent phase diagram calculation, which is required to obtain phase fraction and concentration, has to be repeated during the main calculation. This repetition can be avoided if the mapping file is created in the axes of temperature and average composition for the liquid fraction and the liquid concentration based on the lever rule. Once the mapping file is obtained, only a simple interpolation procedure is needed during the main calculation. This technique is proposed in the present article and explained in Section II, where the macrosegregation and microscopic models are described as well. Numerical implementation is given in Section IV, and the calculation results are discussed in Section V. Section VI summarizes the results and presents conclusions.

\section{MODEL DESCRIPTION}

The macrosegregation model consists of three parts: a macroscopic transfer model, a microscopic model, and a phase diagram calculation module based on the mapping technique. They are described in the following sections.

\section{A. Macroscopic Transfer Model}

The model originally formulated by Bennon and Incropera $^{[4]}$ and reassessed by Prescott and Incropera ${ }^{[10]}$ is adopted as the macroscopic description to predict macrosegregation during DC casting of multicomponent alloys. As is done in most of the studies on this subject, ${ }^{[7,11,12]}$ turbulence in the liquid pool of the billet is neglected because its contribution to the overall macrosegregation pattern is small, whereas the increase in the computation time is very large. Table I summarizes the mathematical formulation of the rigid network model.

The closure of the set of conservation equations listed in Table I requires supplementary relationships for phase mass fraction and composition, which can be derived from microscopic-scale consideration. In this

Table I. Main Equations that Comprise the Macrosegregation Model used in this Work

Mass conservation

Momentum conservation

Heat conservation

Species conservation

Auxiliary relations

$$
\frac{\partial \rho_{m}}{\partial t}+\nabla \cdot\left(\rho_{m} \vec{V}_{m}\right)=0
$$

$$
\begin{aligned}
\begin{aligned}
\frac{\partial\left(\rho_{m} \vec{V}_{m}\right)}{\partial t}+\nabla \cdot\left(\rho_{m} \vec{V}_{m} \vec{V}_{m}\right)= & -\nabla p_{l}+\nabla \cdot\left(\mu_{l} \frac{\rho_{m}}{\rho_{l}} \nabla \vec{V}_{m}\right)-\frac{\mu_{l} \rho_{m}}{K \rho_{l}}\left(\vec{V}_{m}-\vec{V}_{\text {cast }}\right) \\
& -\rho_{l}\left(\beta_{T, l}\left(T-T_{0}\right)+\sum_{i=1}^{n} \beta_{C, l}^{\mathrm{i}}\left(C_{l}^{\mathrm{i}}-C_{0}^{\mathrm{i}}\right)\right) \vec{g}, \\
K=K_{0} \frac{\left(1-f_{s}\right)^{3}}{f_{s}^{2}}[16] &
\end{aligned}
\end{aligned}
$$

where $\mathrm{n}$ is the number of alloying elements.

$$
\frac{\partial\left(\rho_{m} H_{m}\right)}{\partial t}+\nabla \cdot\left(\rho_{m} H_{m} \vec{V}_{m}\right)=\nabla \cdot\left(\lambda_{m} \nabla T\right)-\nabla \cdot\left(\rho_{m}\left(H_{l}-H_{m}\right)\left(\vec{V}_{m}-\vec{V}_{\text {cast }}\right)\right)
$$

$$
\begin{aligned}
& \frac{\partial\left(\rho_{m} C_{m}^{\mathrm{i}}\right)}{\partial t}+\nabla \cdot\left(\rho_{m} C_{m}^{\mathrm{i}} \vec{V}_{m}\right)=\nabla \cdot\left(\rho_{m} f_{l} D_{l}^{\mathrm{i}} \nabla C_{m}^{\mathrm{i}}\right) \\
& \quad+\nabla \cdot\left(\rho_{m} f_{l} D_{l}^{\mathrm{i}} \nabla\left(C_{l}^{\mathrm{i}}-C_{m}^{\mathrm{i}}\right)\right)-\nabla \cdot\left(\rho_{m}\left(C_{l}^{\mathrm{i}}-C_{m}\right)\left(\vec{V}_{m}-\vec{V}_{\text {cast }}\right)\right)
\end{aligned}
$$

$$
\begin{array}{ll}
\rho_{m}=g_{s} \rho_{s}+g_{l} \rho_{l} & H_{m}=f_{s} H_{s}+f_{l} H_{l} \\
1=g_{s}+g_{l} & C_{m}^{i}=f_{s} C_{s}^{\mathrm{i}}+f_{l} C_{l}^{\mathrm{i}} \\
f_{l}=\frac{g_{l} \rho_{l}}{\rho_{m}} & c_{p m}=f_{s} c_{p s}+f_{l} c_{p l} \\
f_{s}=\frac{g_{s} \rho_{s}}{\rho_{m}} & \lambda_{m}=g_{s} \lambda_{s}+g_{l} \lambda_{l} \\
\vec{V}_{m}=f_{s} \vec{V}_{s}+f_{l} \vec{V}_{l} & H_{s}=c_{p s} T \\
& H_{l}=c_{p s} T_{\text {Sol }}+c_{p l}\left(T-T_{\text {Sol }}\right)+L
\end{array}
$$


article, these relationships for multicomponent alloys are obtained based either on the lever rule or on the Gulliver-Scheil equation, and they will be given in more detail in Section B.

The macrosegregation model was implemented using the commercial software CFX, with the exception of the additional advection-like source term in the enthalpy and the solute transfer equation. These terms were implemented within a custom version of the CFX-5.7.1 solver. For the advection term, the high-resolution scheme is employed. The details about the numerical scheme can be found in the CFX-5 Solver Theory Manual. The use of the CFX software offers the advantages of unstructured meshing and parallel computing, which are essential for the application to large-scale DC casting. Unstructured meshing allows good spatial resolutions without increasing the mesh number, which is important for the accuracy of calculations, especially in areas such as the place close to the liquidus line, where chemical inhomogeneities are initiated and the relative movement between the liquid and solid is present. ${ }^{[13]}$

\section{B. Microscopic Model}

Essential for the extension of the macrosegregation model to multicomponent alloys is the description of the solidification path for a multicomponent alloy, i.e., a microscopic model relating the specific enthalpy and local average solute concentration variations to the solid fraction, temperature, and solute concentrations in the solid and liquid parts of the two-phase volume elements. For a given small volume subject to heat extraction and transport of species in the mushy zone, the following equations can be written to obtain the solidification paths assuming that the enthalpy and average concentration variation rate, $\dot{H}$ and $\dot{C}^{\mathrm{i}}$, are known from solving the macroscopic transport equations.

$$
\begin{aligned}
\dot{H} & =c_{p} \frac{\partial T}{\partial t}+L \frac{\partial f_{s}}{\partial t} \\
\dot{C}^{i} & =\dot{f}_{s} C_{s}^{i}+\dot{f}_{l} C_{l}^{i}+f_{s} \dot{C}_{s}^{i}+f_{l} \dot{C}_{l}^{i} \\
1 & =f_{s}+f_{l}
\end{aligned}
$$

Although $\dot{H}$ and $\dot{C}^{\mathrm{i}}$ can be obtained by solving the conservation equations in Table I, it has to be mentioned that in order to be able do that, Eq. [6] has to be included to close the conservation equations, and these two sets of equations have to be solved simultaneously. This is the so-called micro-macro coupling. In our calculations, the Newton-Raplson iteration has been adopted to solve this problem.

The set of Eq. [6] is written for a system where the solid and liquid phases have the same densities and specific heats. It has no consequences for other calculations performed in this article, where these properties are different for different phases.

The primary phase and the liquid phase, along with all other phases precipitated later during solidification, are included in the phase equilibrium calculation.
Thus, the effects of the precipitation of secondary phases on the liquid fraction and the liquid concentration are taken into account on the microscopic scale. On the macroscopic scale, when the conservation equations are solved, the secondary phases are treated together with the primary phase as a single solid phase, so no distinction between the different solid phases is made. It is also assumed that the precipitated secondary phases have the same physical properties as the primary phase, following Reference 6.

In order to solve this set of equations and to obtain the local solidification path, two supplementary relations have to be determined. The first relation describes the dependence of the solid fraction on temperature and average concentration, and the other gives the relation between the average solid and liquid phase compositions. In general, these two relations have to be deduced based on the following conditions:

(1) the assumption of local equilibrium at the solid-liquid interface, and

(2) by solving solute diffusion in the solid and liquid on the scale of grain size/dendrite arm upon typical DC casting conditions.

These conditions, together with the solute balance equation at the interface, enable us to deduce the interface velocity, and thus, the first required supplementary relation is obtained. The other supplementary relation between the average concentration in the solid and liquid phases can be obtained based on the second condition.

Models with different levels of complexity, ranging from a linear approximation of the solute profile in the dendrite arms to the fully numerical solution of the diffusion equations with moving boundary, have been developed to obtain these relations. ${ }^{[8,14,15]}$ In our implementation, the microscopic module is a stand-alone application program that is coupled with THERMO-CALC* to deal with

* THERMO-CALC is a trademark of Thermo-Calc Software AB, Stockholm.

multicomponent alloys. Their coupling with the macroscopic transfer module is based on the mapping technique discussed in Section C. The solidification path of a ternary alloy derived with the lever rule has been described in detail in References 6 and 7. The Gulliver-Scheil model formulation is given in Reference 16. However, instead of truncating the liquid fraction when it is less than a given threshold, the assumption made in Reference 17 is adopted, which assumes an equilibrium state in the remaining interdendritic liquid as soon as the liquidus of a secondary phase has been reached. The full set of equations is given in Table II.

As discussed in Reference 18, remelting occurring during DC casting may bring difficulties, because the local equilibrium assumption does not hold at the solidliquid interface owing to back-diffusion in the solid. Therefore, it has been proposed by Rappaz and Voller that, during remelting, the solid concentration at the 
Table II. Supplementary Relationships Required for the Closure of the Macroscopic Conservation Equations

$$
\begin{aligned}
& T>T_{\mathrm{Liq}}\left(C_{m}^{\mathrm{i}}\right), f_{l}=1, C_{l}^{\mathrm{i}}=C_{m}^{\mathrm{i}} \\
& T_{\mathrm{Liq}} \geq T \geq T_{\mathrm{Sol}},\left\{\begin{array}{l}
C_{m}^{\mathrm{i}}=\left(1-f_{l}\right) k^{\mathrm{i}} C_{l}^{\mathrm{i}}+f_{l} C_{l}^{\mathrm{i}} \\
T=T_{\mathrm{Liq}}\left(C_{l}^{\mathrm{i}}\right)
\end{array}\right. \\
& T<T_{\mathrm{Sol}}\left(C_{m}^{\mathrm{i}}\right), f_{l}=0, C_{l}^{\mathrm{i}}=C_{m}^{\mathrm{i}} / k^{\mathrm{i}}
\end{aligned}
$$

$$
\begin{aligned}
& T>T_{\mathrm{Liq}}\left(C_{m}^{\mathrm{i}}\right), f_{l}=1, C_{l}^{\mathrm{i}}=C_{m}^{\mathrm{i}} \\
& T_{\text {Liq }} \geq T>T_{\text {Eut }},\left\{\begin{array}{l}
C_{m}^{\mathrm{i}}=\int_{0}^{1-f_{l}} k^{\mathrm{i}} C_{l}^{\mathrm{i}}\left(f_{s}^{\prime}\right) d f_{s}^{\prime}+f_{l} C_{l}^{\mathrm{i}} \\
T=T_{\mathrm{Liq}}\left(C_{l}^{\mathrm{i}}\right)
\end{array}\right. \\
& T \leq T_{\text {Eut }},\left\{\begin{array}{l}
f_{l}=\max \left(f_{l}\left(T_{\text {Eut }}^{+}\right)+R_{E}\left(T-T_{\text {Eut }}\right), 0.0\right) \\
C_{l}^{\mathrm{i}}=C_{\text {Eut }}^{\mathrm{i}}
\end{array}\right.
\end{aligned}
$$

interface is not given by the equilibrium condition but by the previous, "frozen" profile. ${ }^{[18]}$ This means that the entire solid concentration profile has to be stored, which increases the demand for computational memory. A simplified alternative used in the current article is that the solid concentration profile can be adjusted instantaneously to the average local concentration changes to an extent that this open system behaves as a close system with the same local composition solidifying in the Scheil mode. This means that the varied part of the average solute, which is usually very small, diffuses sufficiently so that this open system is like a close system with the same average concentration and temperature. Therefore, for this open system, its solid solute profile along dendrite arms still can be calculated based on the Gulliver-Scheil equation. This approximation still keeps the conservation of the solute. It implies that both the solid and the liquid phases in the open system feel the macroscopic variation in an average way and adjust their fractions and concentration profiles immediately, while in the previous approach, ${ }^{[18]}$ the variation is felt by the liquid only, and the solid is affected by adapting its interface portion to the liquid phase.

\section{Mapping Technique}

To test the feasibility of our idea proposed in Section I that the mapping file is created in terms of average concentration and temperature for liquid concentration and fraction, the following estimation was made. For a given $(n+1)$-ary alloy with a nominal composition of $C_{m}$, we assume that its relative segregation is between 15 pct and +15 pct and its solidification range is $100 \mathrm{~K}$. Let us have 100 nodes in resolving the solid range $(1 \mathrm{~K}$ per node) and 15 nodes in the variation range of the average concentration of alloying element $\mathrm{i}\left(0.02 C_{m}^{\mathrm{i}}\right.$ per node). The total line number in this mapping file is equal to

$$
\begin{aligned}
\frac{\Delta T}{1 K / \text { node }} & \times \frac{0.3 C_{m}^{1}}{0.02 C_{m}^{1} / \text { node }} \times \frac{0.3 C_{m}^{2}}{0.02 C_{m}^{2} / \text { node }} \\
& \times \cdots \frac{0.3 C_{m}^{n}}{0.02 C_{m}^{n} / \text { node }}=100 \times 15^{n}
\end{aligned}
$$

The total line number, total variable number, and the memory ( 8 bytes per variable) required are shown in Figure 1. This strategy will work well for ternary and quaternary alloys. Some optimizations have to be used for quinary alloys, and difficulties are expected in sixcomponent alloys under the currently readily available configuration of a personal computer (100 GB hard disk and 2 GB memory). Further improvement of this strategy could be the employment of nonuniform mapping steps.

For a model with a more advanced description of solidification kinetics, the mapping files tabulated based on the lever rule can also be used in the so-called interface cell. ${ }^{[17]}$ Another benefit of the strategy proposed previously is that it is quite easy to switch the solidification path from the lever rule to the GulliverScheil equation, which would only require the compilation of mapping files based on the latter approximation. As discussed in Reference 18, the Gulliver-Scheil equation gives a better description of the real and nonequilibrium solidification path than the lever rule. In Reference 19, the coupling of a multiphase solidification simulation with a multicomponent phase diagram was carried out. They linked the macroscopic variables,

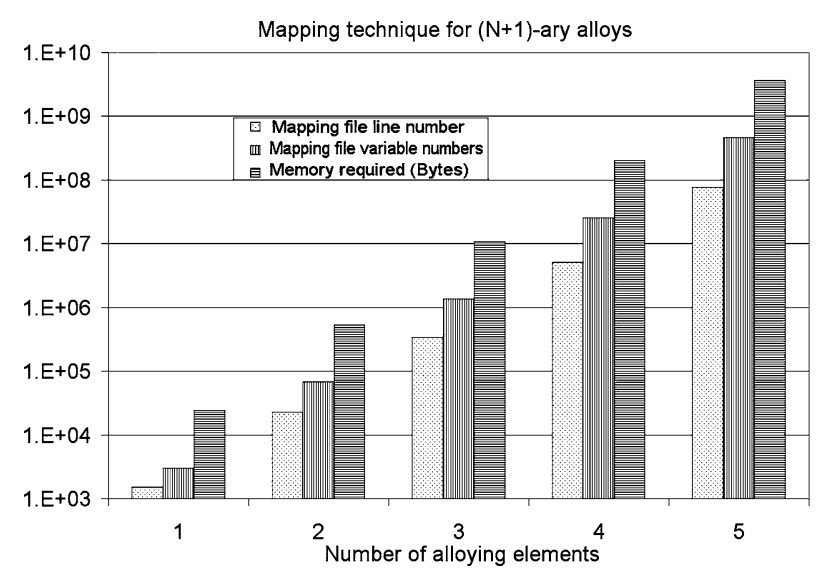

Fig. 1-Schematic graph for showing the requirements for the mapping technique. 
i.e., the average liquid concentration to the microscopic variables (i.e., the concentrations of the solid and liquid phases at the interface). This link can also be built with the mapping technique because there is a unique corresponding relation between the macroscopic variable and the microscopic one.

\section{NUMERICAL IMPLEMENTATION}

The model is applied to a round billet $200 \mathrm{~mm}$ in diameter and $453 \mathrm{~mm}$ in length. The length of the hot top (only its ceramic part embedded in the mold is modeled), mold, air gap, and impingement zone are 20, 15,10 , and $8 \mathrm{~mm}$, respectively, as schematically shown in Figure 2. The settings for the billet and mold dimensions are identical to those used in the experiments described elsewhere. ${ }^{[20]}$ An axisymmetric two-dimensional (2-D) model was used in this work. The 2-D calculation domain used in the reported calculations consisted of 66,583 unstructured cells with a minimum edge size of $1 \mathrm{~mm}$ and a maximum size of $2 \mathrm{~mm}$. The dependence of the calculation results on the mesh size was also evaluated, and the results (not given in this article) showed that the solution is independent on the mesh size used.

The melt of an Al-3.5 wt pet Cu-1.5 wt pet $\mathrm{Mg}$ alloy at a temperature of $997 \mathrm{~K}$ enters from the inlet at the casting speed $120 \mathrm{~mm} / \mathrm{min}$ multiplied by the ratio between the densities of the solid and liquid phases. At the outlet, the solid leaves the domain at the casting speed. A no-slip flow boundary condition is applied on all walls. To make the solid, which adheres to the walls of the mold, air gap, impingement, and water film domains, to have a velocity equal to the casting speed, the velocities of the walls of all mentioned domains are intentionally set to be equal to the casting speed. The boundary condition for the heat-transfer equation is as follows: at the inlet, the melt enters at the casting temperature; and, at the outlet, an adiabatic boundary condition is applied. The hot top does not extract any heat, but the chill mold, air gap, impingement zone, and water film zones do. The heat fluxes on these walls are calculated by heat-transfer coefficients, which are 5000 , $2500,10,000$, and $10,000 \mathrm{~W} \mathrm{~m}^{-2} \mathrm{~K}^{-1}$, respectively. These boundary conditions, together with the mesh used, are shown in Figure 2. Other input parameters required for this model are related to thermodynamic and physical properties of this alloy and are listed in Table III. For the sake of simplicity, the diffusion coefficients of the species are taken as equal, because they are insignificant in determining the final segregation pattern. Calculations were performed for steady-state conditions.

Five cases were considered, as summarized in Table IV.

The hypothetical pseudo-binary system section of the ternary $\mathrm{Al}-\mathrm{Cu}-\mathrm{Mg}$ phase diagram mentioned in case 1 is shown in Figure 3. The partitioning of $\mathrm{Mg}$ at the microscopic scale is considered, but the macroscopic solute transfer is not. Therefore, there is an influence of $\mathrm{Mg}$ on the solidus and liquidus lines, but there is no macroscopic segregation of this element; its average concentration is fixed at the nominal value. For Gulliver-Scheil type solidification, the $\mathrm{Cu}$ concentration of the liquid phase follows the liquidus line shown in Figure 3, and the $\mathrm{Cu}$ concentration of the solid phase at the solid-liquid interface follows the solidus line. This solidification path reflects the hypothetical ternary alloy where only one of the solutes segregates.

In cases 2 through 4 , the solidification paths of this alloy are according to the full ternary phase diagram. The two solidification paths, based on the lever rule and Gulliver-Scheil equation, respectively, are drawn on the projected liquidus surface of the ternary $\mathrm{Al}-\mathrm{Cu}-\mathrm{Mg}$

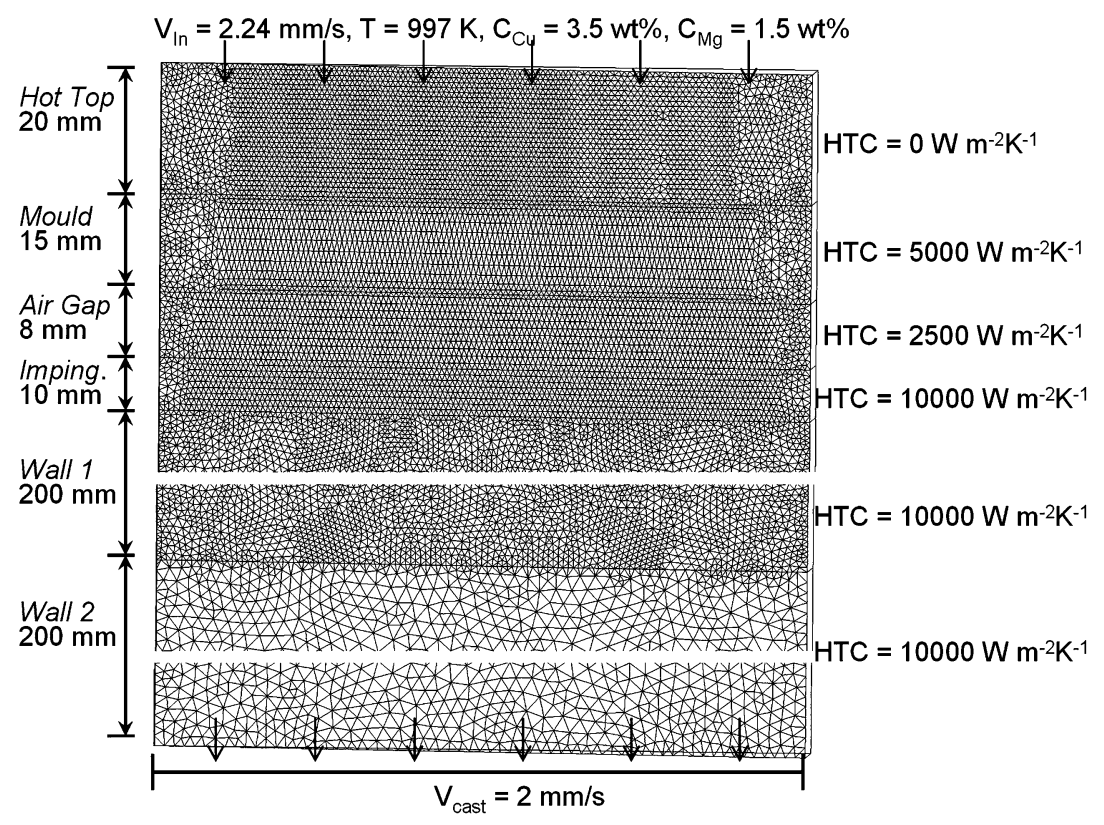

Fig. 2-Mesh used and the boundary conditions imposed. 
Table III. Thermodynamic and Physical Properties of an Al-3.5 Wt Pet Cu-1.5 Wt Pet Mg Alloy ${ }^{[21,22,23]}$

\begin{tabular}{llcl}
\hline Property & \multicolumn{1}{c}{ Values } & Property & \multicolumn{1}{c}{ Values } \\
\hline$\mu_{l}$ & $0.0013 \mathrm{~kg} \mathrm{~m}^{-1} \mathrm{~s}^{-1}$ & $c_{l}$ & $1054 \mathrm{~J} \mathrm{~kg}^{-1} \mathrm{~K}^{-1}$ \\
$\rho_{l}$ & $2460 \mathrm{~kg} \mathrm{~m}^{-3}$ & $\lambda_{l}$ & $95 \mathrm{~W} \mathrm{~m}^{-1} \mathrm{~K}^{-1}$ \\
$\beta_{T, l}$ & $1.17 \times 10^{-4} \mathrm{~K}^{-1}$ & $\rho_{s}$ & $2750 \mathrm{~kg} \mathrm{~m}^{-3}$ \\
$\beta_{\mathrm{Cu}, l}$ & 0.73 & $c_{s}$ & $958 \mathrm{~J} \mathrm{~kg}^{-1} \mathrm{~K}^{-1}$ \\
$\beta_{\mathrm{Mg}, l}$ & -0.41 & $\lambda_{s}$ & $180 \mathrm{~W} \mathrm{~m}^{-1} \mathrm{~K}^{-1}$ \\
$D_{l}^{\mathrm{Cu}}$ & $3 \times 10^{-9} \mathrm{~m}^{2} \mathrm{~s}^{-1}$ & $L$ & $3.9 \times 10^{5} \mathrm{~J} \mathrm{~kg}^{-1}$ \\
$D_{l}^{\mathrm{Mg}}$ & $3 \times 10^{-9} \mathrm{~m}^{2} \mathrm{~s}^{-1}$ & $K_{0}$ & $6.67 \times 10^{-11} \mathrm{~m}^{2}$ \\
\hline
\end{tabular}

phase diagram in Figure 4. For the lever-rule type solidification, the solidification ends at a higher temperature and there is no secondary phase formed. While in the case of the Gulliver-Scheil type solidification, the solidification path hits the monovariant line. Afterward, as the solidification proceeds, the $\mathrm{Cu}$ concentration in the liquid phase keeps increasing while the $\mathrm{Mg}$ concentration in the liquid phase decreases, which is a unique characteristic that is not present in the binary case. Finally, the solidification ends at the ternary eutectic point. A mapping file contains several thousand of these kinds of paths for a wide range of composition so that it gives a full description of the solidification paths encountered in the calculations.

As described in Section II, the mushy zone permeability is assumed to be isotropic and is evaluated using the Blake-Kozeny expression. In cases 1 through 4, the permeability coefficient, $K_{0}$, which is proportional to the square of dendrite arm spacing, is set to $6.67 \times 10^{-11} \mathrm{~m}^{2}$, as in Reference 11 . In case 5 , the permeability is $5 \times 10^{-10}$, while the rest of the parameters in this calculation are the same as in case 4 . As discussed in Reference 12, the permeability of the mushy zone may determine the sign and the extent of the centerline segregation. The motivation of performing case 5 is to have better agreement with the measured experimental segregation profile by tuning the permeability coefficient.

\section{RESULTS AND DISCUSSION}

Cases 1 and 2 are examined for parametric study purposes, and, artificially, solidification shrinkage is not taken into account. It is intended that by comparing these two cases, the effect of the solute $\mathrm{Mg}$ to the overall macrosegregation profile could be revealed. The solute transfer equation for $\mathrm{Mg}$ has been solved in case 2 and has not been solved in case 1. As has been concluded in the parametric study in Reference 21, solutal buoyancy is more effective than thermal buoy-

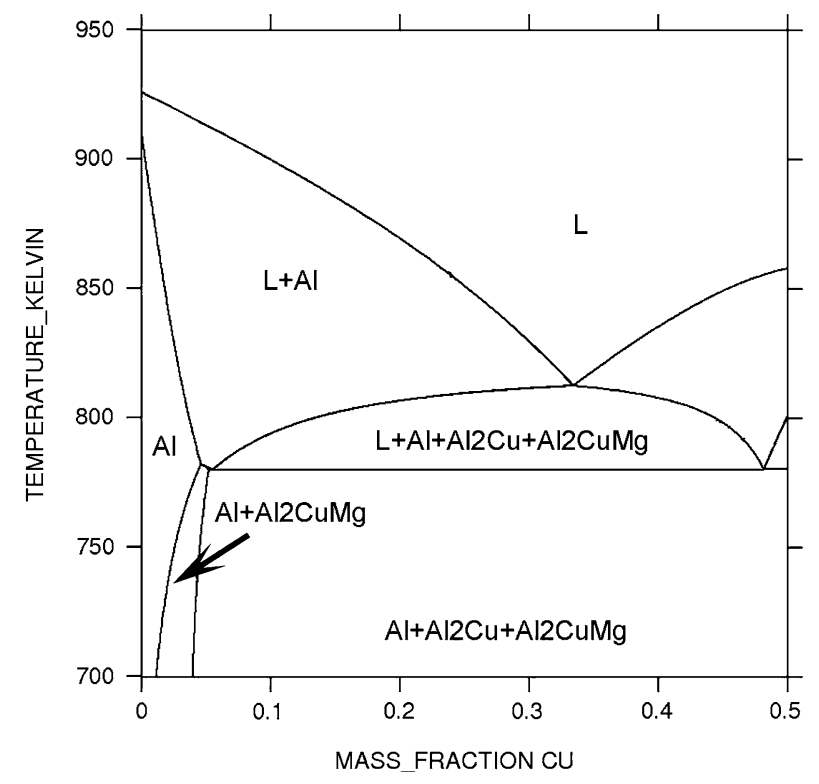

Fig. 3-Vertical section of the ternary $\mathrm{Al}-\mathrm{Cu}-\mathrm{Mg}$ phase diagram with the weight fraction of $\mathrm{Mg}$ equal to $1.5 \mathrm{wt}$ pct, calculated by THERMO-CALC.

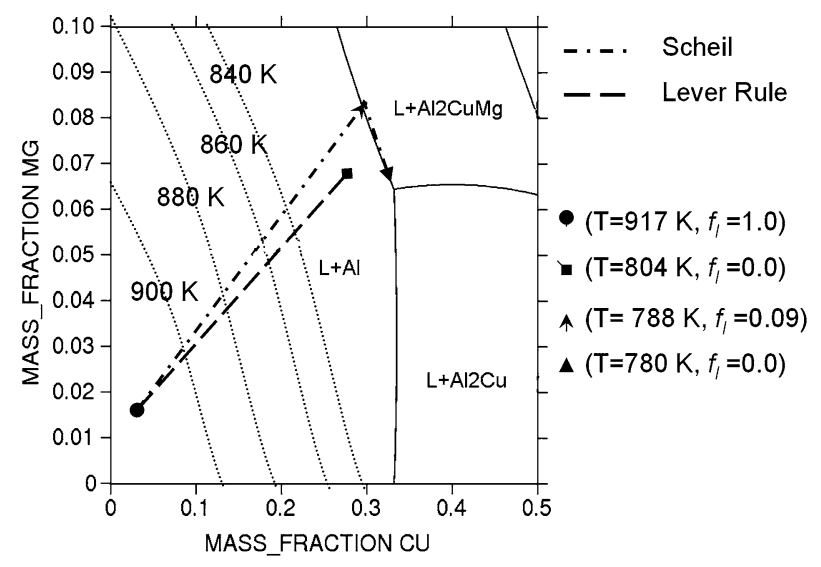

Fig. 4 -Solidification paths for an Al-3.5 wt pet $\mathrm{Cu}-1.5$ wt pet $\mathrm{Mg}$ alloy based on the lever rule or the Gulliver-Scheil equation, calculated by THERMO-CALC.

ancy in augmenting the flow that brings the solute to the center and tends to form positive segregation there. Therefore, this solutal buoyancy caused by the addition of a second alloying element, which is only present in case 2, may make some difference in the final segregation pattern. Due to the fact that the liquid phase density is inversely linked to the concentration of $\mathrm{Mg}$,

Table IV. Main Characteristics of Calculations Performed in the Present Work

\begin{tabular}{|c|c|}
\hline Case 1 & $\begin{array}{l}\text { no solidification shrinkage, thermosolutal buoyancy, and Gulliver-Scheil equation with a hypothetical pseudo-binary } \\
\text { system section }\end{array}$ \\
\hline Case 2 & no solidification shrinkage, thermosolutal buoyancy, and Gulliver-Scheil equation with the ternary phase diagram \\
\hline Case 3 & solidification shrinkage, thermosolutal buoyancy, and lever rule with the ternary phase diagram \\
\hline Case 4 & solidification shrinkage, thermosolutal buoyancy, and Gulliver-Scheil equation with the ternary phase diagram \\
\hline Case 5 & The same as case 4 , but with a higher permeability constant \\
\hline
\end{tabular}


it is expected that the extent of macrosegregation of $\mathrm{Cu}$ in case 2 would be less than in case 1 . Results shown in Figure 5 confirm this estimation. Both cases 1 and 2 predict positive centerline segregation, and in case 2, the relative segregation of $\mathrm{Cu}$ is $1.7 \mathrm{pct}$, which is about 20 pct less than in case 1 . This alleviation in macrosegregation in case 2 is caused directly by the fact that the flow beneath and along the liquidus contour and upward flow at the center in case 2 is weaker than in case 1. Figure 6 illustrates the contribution of solutal buoyancy to the flow pattern in the billet sump by the velocity difference, which is calculated by the velocity field in case 1 minus that in case 2 . This velocity difference is more detectable close to the center of the billet with a magnitude of $0.8 \mathrm{~mm} / \mathrm{s}$. Consistently, it is the place where the maximum difference in average concentrations occurs.

The small variation of the relative concentrations in the outer part of the billet between 0.06 and $0.09 \mathrm{~m}$ along the radius, as shown in Figure 5, are apparently linked to a peculiar flow pattern (vortices) that appears close to the coherency isotherm in this section of the billet. It is premature to discuss if these oscillations are physical. More experimental and numerical work is needed.

Cases 3 and 4 take into account solidification shrinkage. In the center of the billet, negative segregation is predicted in these two calculations, as illustrated in Figure 7.

Previously, we have shown that shrinkage-induced flow occurs in the deep part of the mushy zone, i.e., beneath the coherency solid fraction contour, estimated in Reference 21 as 0.2 . This shrinkage-induced flow is almost perpendicular to the solid fraction contour and is directed to the deeper part of the mushy zone with the magnitude at a scale of $0.1 \mathrm{~mm} / \mathrm{s}$. There are two components in this flow: one is horizontal along the radius directed toward the billet surface and the other is vertical along the casting direction. Although the vertical downward flow will dilute the local volume element by bringing less enriched liquid into it, the negative centerline segregation will not form if only this vertical downward flow is present, because as solidification proceeds, the process eventually comes to the point when less and less solute is taken out and only solute accumulation occurs. This case is identical to the steady-state unidirectional solidification analyzed by Flemings and Nereo. ${ }^{[1]}$ It is the horizontal component that takes the solute away from the center to the surface. ${ }^{[12,24,25]}$ The depletion in the center cannot be compensated, as there is no horizontal inflow of the solute from more enriched regions. At the surface, there is a pileup of the solute as there is no outflow. Therefore, there is an overall solute transfer from the center of the billet to its surface. Because the magnitude of the induced flow is mainly dependant on the shrinkage ratio, one may conclude that macrosegregation can be reduced by making the solid fraction contour flatter so that the horizontal component of the shrinkage-induced flow will be smaller. On the other hand, all the measures that cause a steeper solid fraction isoline, such as increasing the casting speed, will lead to more severe negative centerline segregation resulting from shrinkageinduced flow. A more careful treatise of the relationship between the macrosegregation and the steepness of the coherency isotherm has been reported in a separate article. ${ }^{[26]}$

Case 4, based on the Gulliver-Scheil approximation, predicts more severe segregation than case 3 based on the lever rule, highlighting the importance of having a good description of the solidification path of a multicomponent alloy when solidification shrinkage is present. The severe segregation in case 4 can be easily understood by examining the solidification paths shown in Figure 4, because the liquid phase is much more enriched upon Gulliver-Scheil type solidification. Therefore, under the same flow condition caused by solidification shrinkage in the deep part of the mushy zone, there is more transferred solute in case 4 than in case 3 .

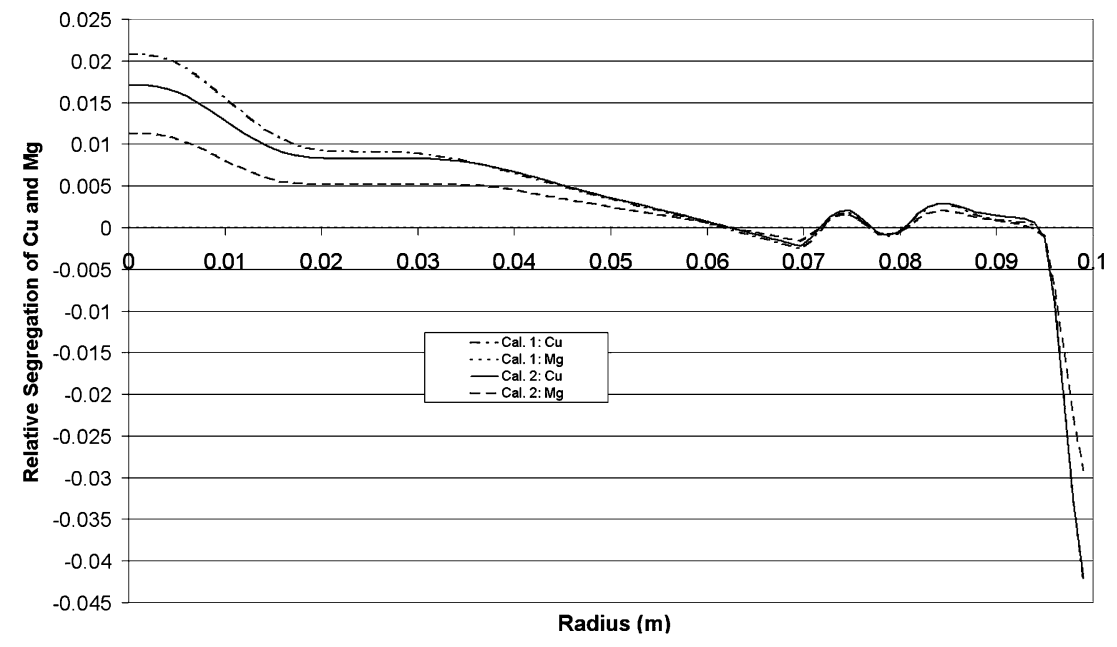

Fig. 5-Relative segregation of $\mathrm{Cu}$ and $\mathrm{Mg}\left[C_{m}-C_{0}\right] / C_{0}$ along the radius from the center (on the left) to the surface (on the right) of a billet cast at $120 \mathrm{~mm} / \mathrm{min}$; cases 1 and 2 are explained in Table IV. Note that the relative segregation of $\mathrm{Mg}$ obtained in case 1 is zero. 


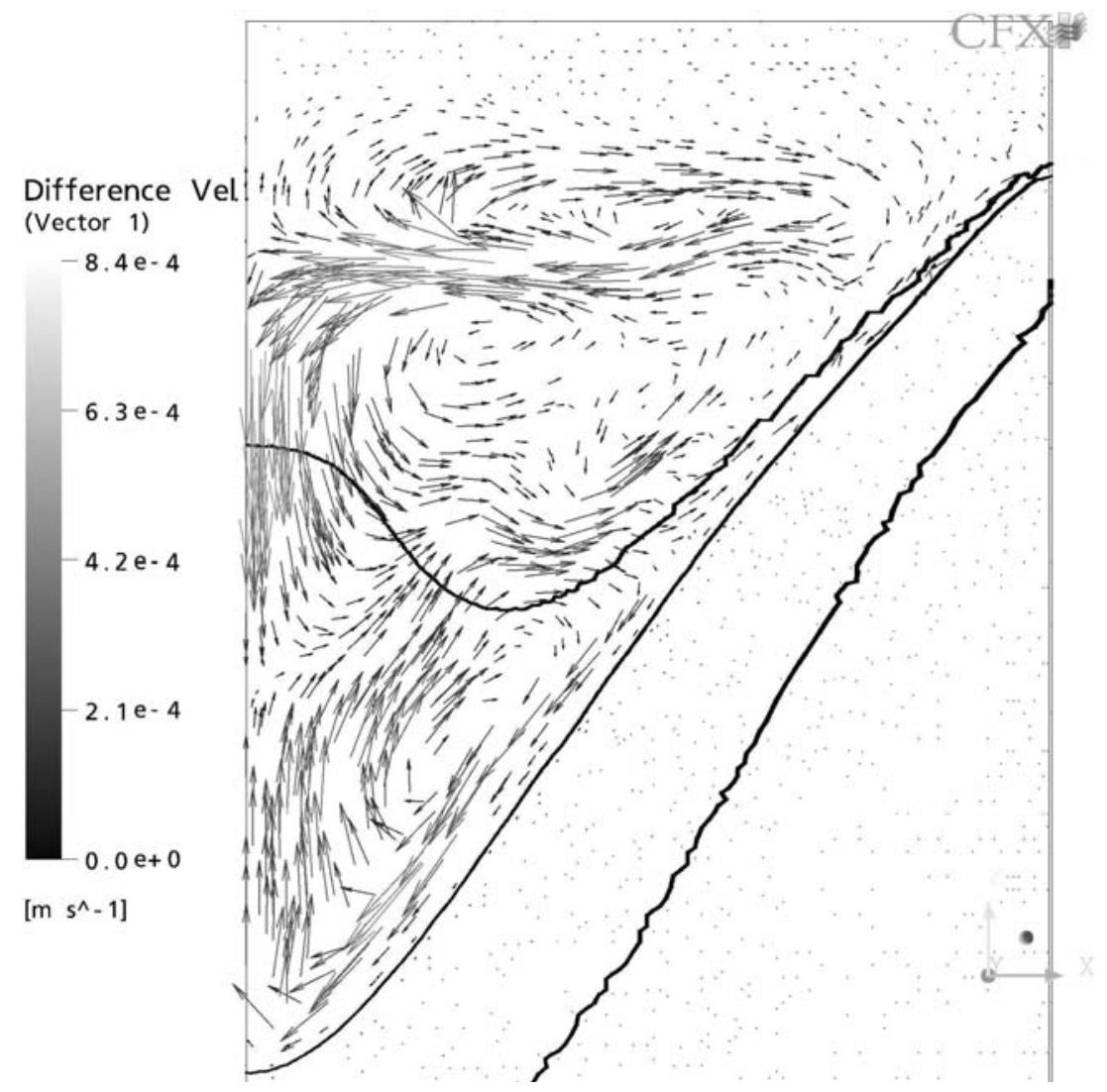

Fig. 6-Velocity difference calculated by the velocity field in case 1 minus that in case 2 .

The comparison of the calculated results in Figure 7 with experimental values in Figure 8 (a) shows that, although the qualitative agreement is found in terms of the general segregation pattern and the difference in $\mathrm{Mg}$ and $\mathrm{Cu}$ segregation, the predicted negative centerline segregation is overestimated.

There are many factors that could contribute to this overestimation such as the values of physical properties used and the assumptions made in the model. However, a permeability model is believed to be an important contributor due to the difficulties in measuring the permeability and to the assumption made in the model (the rigid network model is not accurate when the solid fraction is low). To obtain better agreement with the experimental results, case 5 , in which the permeability constant was increased by a factor of 7.5 to $5 \times 10^{-10}$, was considered. The results presented in Figure 8(b) show some improvement as compared with the calculation results shown in Figure 7. However, this agreement is based on the higher permeability constant, i.e., the coarser microstructure, because the permeability constant is proportional to the DAS, according to Eq. [8]: ${ }^{[27]}$

$$
K_{0}=\frac{\lambda_{2}^{2}}{180}
$$

The resultant dendrite arm spacing is $300 \mu \mathrm{m}$. It is much higher than the experimentally measured one, i.e., 20 to $30 \mu \mathrm{m}$. This discrepancy exposes the potential problem in applying the Blake-Kozeny relation to calculate permeability in the range of very low solid fractions. A way to overcome this problem has been suggested by Vreeman et al. in Reference 28; the flow in the range of very low solid fractions is not modeled by the Darcy law but by a simple drag model. This improvement will be explored in our future study and is beyond the scope of this article. At this moment, it is concluded from this confrontation with the experimental data that a semiquantitative agreement is achieved when both thermosolutal buoyancy and shrinkage are taken into account. A better description of the permeability in the slurry zone is needed to have a better agreement with the experimental data.

There are still some differences in segregation profiles between case 5 and the experimental measurement. At the middle of the radius, case 5 predicts rather flat and slightly negative segregation, while the experimental measurement shows relatively large positive segregation. About $1 \mathrm{~cm}$ beneath the surface, there is a negative segregation in the experimental measurement, which is not present in case 5 .

Although both case 5 and the experimental measurement give positive segregation at the surface, the extent in the latter case is much larger. There are a few factors that may affect the segregation pattern but have not been explored in this article, i.e., floating grains, exudation, and possible numerical effects caused by the choice of the mesh. These issues will be the subjects of future work. 


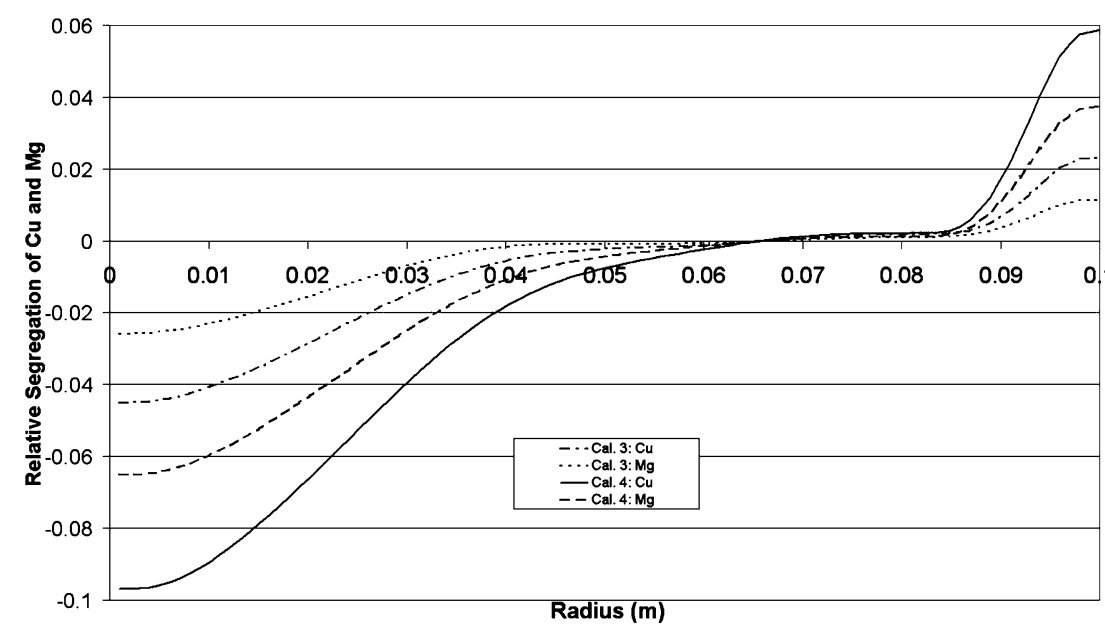

Fig. 7-Relative segregation of $\mathrm{Cu}$ and $\mathrm{Mg}\left[C_{m}-C_{0}\right] / C_{0}$ along the radius from the center (on the left) to the surface (on the right) of a billet. Cases 3 and 4 are explained in Table IV.

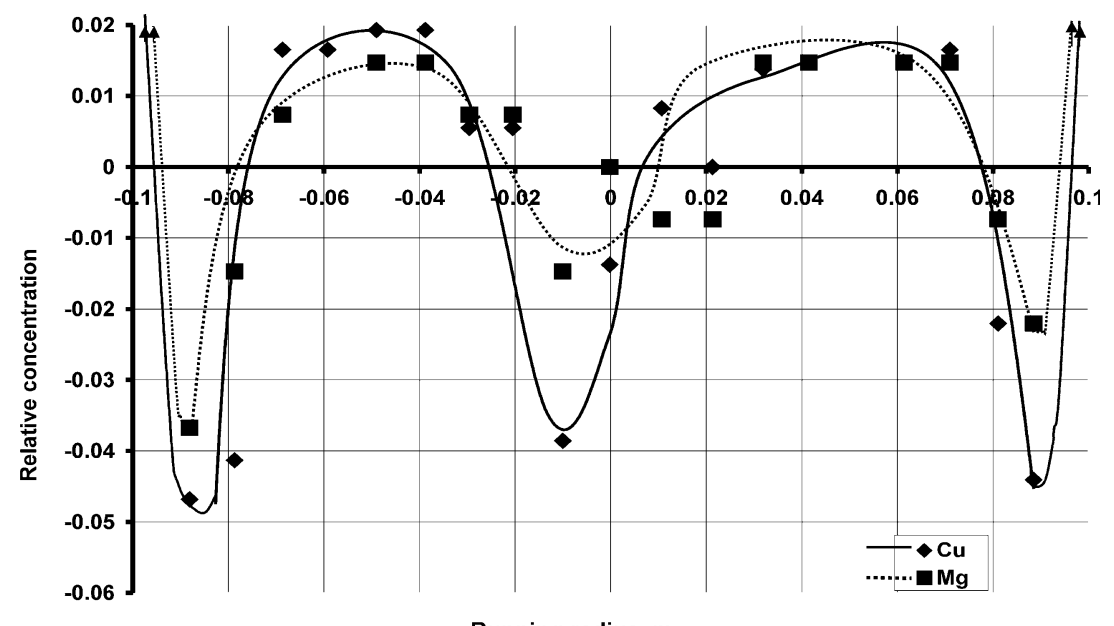

(a)

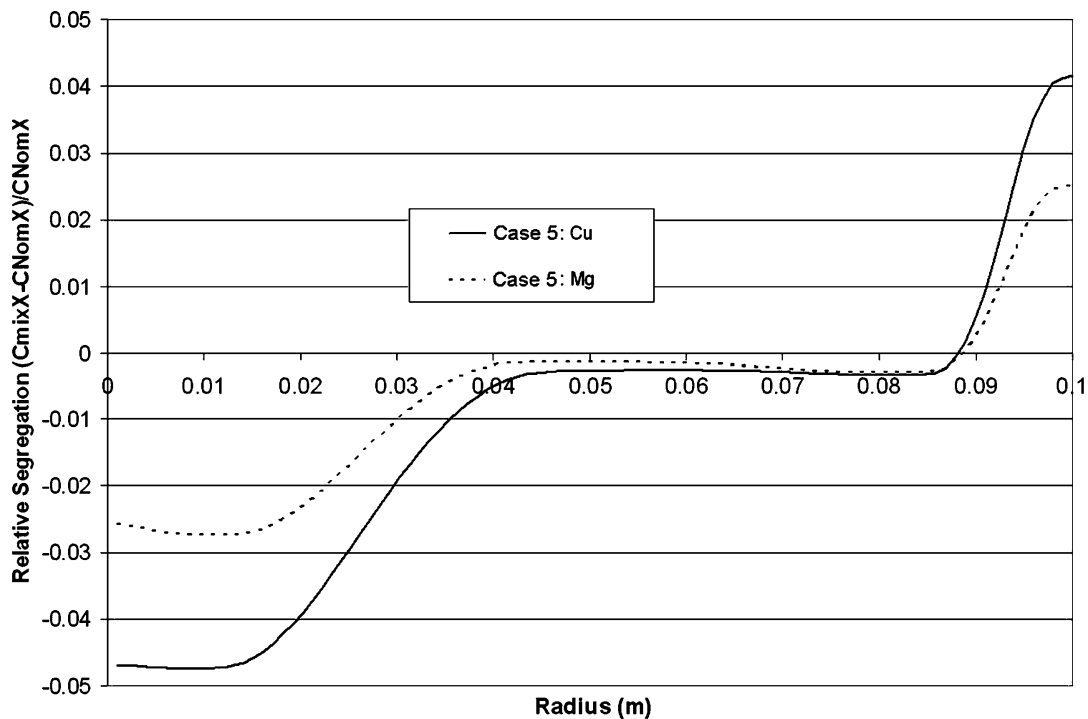

(b)

Fig. 8- (a) Experimentally observed and (b) calculated macrosegregation of $\mathrm{Cu}$ and $\mathrm{Mg}$ in a 200-mm round billet of a grain-refined 2024 (Al-Cu-Mg) alloy cast at $12 \mathrm{~cm} / \mathrm{min}$; case 5 is explained in Table IV. 


\section{SUMMARY AND CONCLUSIONS}

A macrosegregation model is implemented in a commercial CFD code using a modified mapping technique and applied to a ternary $\mathrm{Al}-\mathrm{Cu}-\mathrm{Mg}$ alloy. Our study shows the following.

1. The addition of the secondary alloying element, $\mathrm{Mg}$, will influence the final segregation pattern by its contribution to the solutal buoyancy term. It is important to consider the contribution of every solute to the buoyancy force, as shown in cases 1 and 2; they may have opposite contributions to the overall flow.

2. An appropriate choice of the description of the solidification path is important in predicting the macrosegregation induced by solidification shrinkage, as concluded from the comparison of cases 3 and 4.

3. Semiquantitative agreement with the experimental data is achieved in calculations that take into account both thermosolutal buoyancy and shrinkage. Some overestimation of centerline segregation is obtained, though the model used does not take into account the effect of floating grains on macrosegregation. A better description of the permeability in the mushy zone, solid transport in the slurry zone, and surface exudation is needed to obtain better agreement with experimental data.

\section{NOMENCLATURE}

$c_{p}$
$C$
$D$
$f$
$g$
$H$
$k$
$K$
$p$
$L$
$m_{s}$
$t$
$T$
$T_{\text {Eut }}$
$T_{\text {Liq }}$
$T_{\text {Sol }}$
$V$
$\beta_{C}$
$\beta_{T}$
$\lambda$
$\mu$
$\rho$
$R_{E}$

specific heat $(\mathrm{J} / \mathrm{kg} \mathrm{K})$

mass fraction of alloy element mass diffusion coefficient $\left(\mathrm{m}^{2} / \mathrm{s}\right)$

mass fraction

volume fraction

enthalpy $(\mathrm{J} / \mathrm{kg})$

equilibrium partition coefficient

permeability $\left(\mathrm{m}^{2}\right)$

pressure $\left(\mathrm{N} / \mathrm{m}^{2}\right)$

latent heat $(\mathrm{J} / \mathrm{kg})$

liquidus slope $(\mathrm{K})$

time (s)

temperature $(\mathrm{K})$

eutectic reaction temperature $(\mathrm{K})$

liquidus of composition $C_{m}(\mathrm{~K})$

solidus of composition $C_{m}(\mathrm{~K})$

velocity $(\mathrm{m} / \mathrm{s})$

solutal expansion coefficient

thermal expansion coefficient $(1 / \mathrm{K})$

thermal conductivity $(\mathrm{W} / \mathrm{m} \mathrm{K})$

dynamic viscosity $(\mathrm{kg} / \mathrm{s} \mathrm{m})$

density $\left(\mathrm{kg} / \mathrm{m}^{3}\right)$

eutectic reaction rate $\left(\mathrm{K}^{-1}\right)$

\section{Subscripts}

$l, s$, and $m$ liquid, solid, and mixture, respectively

$\mathrm{i} \quad$ alloying element $\mathrm{i}$

\section{ACKNOWLEDGMENTS}

The authors thank Dr. Ian Hamill and Paul Guilbert for providing a customized version of CFX 5.7.1. Special thanks go to Dr. R. Nadella for experimental data on macrosegregation in a 2024 alloy. This work is performed within the framework of the research program of the Netherlands Institute for Metals Research (www.nimr.nl), Project No. MC4.02134.

\section{REFERENCES}

1. M.C. Flemings and G.E. Nereo: Trans. TMS-AIME, 1967, vol. 239, pp. 1449-61.

2. C.Y. Wang and C. Beckermann: Metall. Mater. Trans. A, 1996, vol. 27A, pp. 2754-64.

3. J. Ni and C. Beckermann: Metall. Trans. B, 1991, vol. 22B, pp. 349-61.

4. W.D. Bennon and F.P. Incropera: Int. J. Heat Mass Transfer, 1987, vol. 30, pp. 2161-70.

5. C. Beckermann: Int. Mater. Rev., 2002, vol. 47 (5), pp. 243-61.

6. M.J.M Krane and F.P. Incropera: Int. J. Heat Mass Transfer, 1997, vol. 40 (16), pp. 3827-35.

7. M.J.M. Krane and F.P. Incropera: Int. J. Heat Mass Transfer, 1997, vol. 40 (16), pp. 3837-47.

8. L. Thuinet and H. Combeau: J. Mater. Sci., 2004, vol. 39, pp. 7213-19.

9. X. Dore, H. Combeau, and M. Rappaz: Acta Mater., 2000, vol. 48, pp. 3951-62.

10. P.J. Prescott and F.P. Incropera: Int. J. Heat Mass Transfer, 1991, vol. 34 (9), pp. 2351-59.

11. C.J. Vreeman, H.J.M. Krane, and F.P. Incropera: Int. J. Heat Mass Transfer, 2000, vol. 43, pp. 687-704.

12. A.V. Reddy and C. Beckermann: Metall. Mater. Trans. B, 1997, vol. 28B, pp. 479-89.

13. T.U. Kaempfer and M. Rappaz: Modelling Simul. Mater. Sci. Eng., 2003, vol. 11, pp. 575-97.

14. C.Y. Wang and C. Beckermann: Metall. Mater. Trans. A, 1996, vol. 27A, pp. 2754-64.

15. H. Combeau, J.-M. Drezet, A. Mo, and M. Rappaz: Metall. Mater. Trans. A, 1996, vol. 27A, pp. 2314-26.

16. Q. Chen and B. Sundman: Mater. Trans., 2002, vol. 43 (3), pp. 551-59.

17. Q. Du and A. Jacot: Acta Mater., 2005, vol. 53, pp. 3479-93.

18. M. Rappaz and V. Voller: Metall. Trans. A, 1990, vol. 21A, pp. 749-53.

19. A. Ludwig, M. Gruber-Pretzler, F. Mayer, A. Ishmurzin, and M. Wu: Mater. Sci. Eng. A, 2005, vol. 413-414, pp. 485-89.

20. D.G. Eskin, J. Zuidema, V.I. Savran, and L. Katgerman: Mater. Sci. Eng. A, 2004, vol. 384, pp. 232-44.

21. Q. Du, D.G. Eskin, and L. Katgerman: in Modelling of Casting, Welding and Advanced Solidification Processes XI, C.-A. Gandin and M. Bellet, eds., TMS, Warrendale, PA, 2006, pp. 235-42.

22. E.A Brandes and G.B Brook: Smithells Metals Reference Book, Butterworth-Heinemann, Oxford, United Kingdom, 1992.

23. A.E. Vol: Handbook of Binary Metallic Systems: Structure and Properties Vol. 1, Israel Program for Scientific Translations, Jerusalem, 1966.

24. V.I. Dobatkin: Continuous Casting and Casting Properties of Alloys, Oborongiz, Moscow, 1948.

25. R. Mehrabian, M. Keane, and M.C. Flemings: Metall. Trans., 1970, vol. 1, pp. 1209-20.

26. D.G. Eskin, Q. Du, and L. Katgerman: Scripta Mater., 2006, vol. 55, pp. 715-18.

27. S. Asai and I. Muchi: Trans. Iron Steel Inst. Jpn., 1978, vol. 18, pp. 90-98.

28. C.J. Vreeman, H.J.M. Krane, and F.P. Incropera: Int. J. Heat Mass Transfer, 2000, vol. 43, pp. 677-86. 